\title{
La gramática en contexto: Una aproximación didáctica a la gramática
}

Yehicy Orduz Novarrete

Profesora área de Lingüistica

Universidad Pedagógica Nacional
... nuestras relaciones con los educandos, a la vez que nos exigen respeto hacia ellos, nos imponen igualmente el conocimiento de las condiciones concretas de su contexto,

que lo condiciona.

Cartas a quien pretenda enseñar

Sexta carta - Paulo Freire

\section{Preámbulo}

a gramática ${ }^{81}$ y sus procesos de enseñanza-aprendizaje han sido por mucho, temas que generan debate en el ámbito académico de la educación básica, media y universitaria. Maestros, estudiantes y docentes en formación reconocen sus ventajas pero en realidad, pocos atinan en descubrir su innegable utilidad y muchos menos, en antojarse de su estudio.

La discusión acerca de su inclusión o exclusión del aula de clase es permanente, no sólo en nuestro país sino en muchos otros alrededor del mundo. Basta sólo con escribir en el buscador gramática - enseñanza, para que se nos muestren millones de documentos, con otro tanto de definiciones, propuestas, discusiones, disquisiciones, encuentros y des-encuentros que, teniendo probabilidad de abordar, sólo nos permitirian ver una pequeña parte del panorama.
Aún es más, no es secreto que el trabajo gramatical genera cierto malestar entre niños, jóvenes y algunos universitarios. Su práctica, con frecuencia repetitiva y abstracta, ha logrado que muchos escuchen el nombre y retornen en su mente a la época de las planas, la memorización, el listado infinito de diga equis y no diga zeta, las vastas columnas de raíces griegas y latinas, el uso de signos ininteligibles, los pesados marcadores de frase, y mil cosas más, bastante alejadas de la realidad. Y sólo hablamos de la lengua materna.

Así, la verdad es que la gramática y sus prácticas no son materia fácil. Su contenido, con frecuencia, más prescriptivo que perceptivo, ha conseguido que ni desde la docencia ni desde el estudio se llegue por completo a un momento feliz en el que pueda verse más allá del precepto y empiece a pensarse, como medio, como excusa para generar conocimiento.

\footnotetext{
${ }^{81}$ Entenderemos como gramática, los dos sentidos, el estricto y el amplio, en los que la entiende la Nueva gramática de la lengua española: "En su sentido más estricto, la GRAMÁTICA estudia la estructura de las palabras, las formas en que estas se enlazan y los significados a los que tales combinaciones dan lugar. En este sentido, la gramática comprende la MORFOLOGía, que se ocupa de la estructura de las palabras, su constitución interna y sus variaciones, y la SINTAXIS, a la que corresponde el análisis de la manera en que se combinan y se disponen linealmente, así como el de los grupos que forman. ... En un sentido más amplio, la gramática comprende, además, el análisis de los sonidos del habla, que corresponde a la FONÉTICA, y el de su organización lingüistica, que compete a la FONOLOGIA". RAE (2010:3).
} 
Como estudiosos del lenguaje y como docentes, estas reflexiones nos han llevado a planteamos, ¿qué estrategia de enseñanza-aprendizaje sería más adecuada para abordar la gramática de la lengua castellana? En las siguientes líneas no vamos a resumir los métodos de enseñanza de la gramática, sería repetitivo. Tampoco vamos a criticarlos, no es nuestro interés. Nos centraremos en hacer algunas reflexiones que nos permitan demostrar que una gramática en contexto es una estrategia pedagógica, no muy novedosa pero si algo olvidada, para lograr que los estudiantes aprendan y sobre todo, se interesen en el análisis de los hechos lingüísticos que revelan ese complejo fenómeno que nos identifica como especie, como seres humanos: el lenguaje. Pero la idea no es quedarnos ahi. Como propuesta, este documento queda a juicio del lector para que, a través de su aplicación en los diversos espacios y niveles educativos, verifique si el interés que con ella se despierte, puede volver a generar conocimiento lingüistico.

\section{Nuestra propuesta}

La propuesta de enseñanza de la gramática en contexto nace desde la experiencia, del afán por despertar el gusto por la cuestión, de cambiar la muy extendida práctica de unos estudios gramaticales regidos por la normatividad y la formalidad, y fundamentados en la escritura. Una crítica muy interesante al respecto puede encontrarse en Mier ${ }^{22}$ (2010:7) quien señala: "la lengua se "enseña" como un sistema de signos aislado del contexto sociocultural del alumno, y su estudio se queda en un inventario de categorías y en la descripción de sus componentes, ejercicio mecánico que poco o nada aporta al análisis crítico que el individuo, a través del lenguaje, debe hacer de su realidad".

De esto ha resultado, además de la aversión, una práctica estéril que, al estar centrada en lo gramaticalmente correcto, deja sin contemplar las adecuaciones contextual y discursiva necesarias para producir y entender discursos. Olvida la realidad.

Nuestra gramática en contexto es todo lo contrario. Al ser una estrategia de enseñanza ${ }^{83}$ con un fuerte cimiento en lo comunicativocognitivo, toma en cuenta la configuración de los universos conceptuales y socio-culturales que producen y permiten comprender el acto de comunicación. Aquí, la gramática se une a la semántica y la pragmática, para ir más allá de lo literal, para explicar en el discurso, los procesos de cognición y significación que establecen las estrategias utilizadas por el hablante para lograr sus propósitos.

Esto claro está, no ha de limitarse al trabajo en aula de clase. La innovación de nuestra propuesta se centra en el trabajo de campo, en las salidas pedagógicas ${ }^{84}$. Siendo que el interés principal es llamar la atención del estudiante,

\footnotetext{
${ }^{82}$ Podemos encontrar un nutrido estudio crítico de los libros de texto colombianos en los trabajos hechos por Freddy Mier Logato, de la Universidad Surcolombiana: Análisis crítico de contenidos, condiciones de producción y usos de los textos de español para bachillerato en Colombia (2009); ¿Qué pasa con la enseñanza de la lengua y el lenguaje? Reflexiones y aportes desde la comunicación y el análisis del discurso (2009); Libros de texto o la limitación de los procesos pedagógicos (2008); entre otros.

${ }^{83}$ Confrontar: Artículos 76 y 77 de la Ley 115 de 1994, Artículo 33 del decreto 1860'de 1994 y Artículo 2 del decreto 230 de 2002 , en donde se invita a innovar las pedagogias y estrategias de enseñanza en los distintos niveles con el objeto de facilitar el aprendizaje y fomentar el conocimiento.

${ }^{84}$ El pilotaje de esta propuesta se llevó a cabo entre 2007 y 2009 con seis salidas pedagógicas del ambiente de Ciencias del lenguaje, espacio académico de Sistemas fonético-fonológicos de la Universidad Pedagógica Nacional. Éstas, tituladas: "Conociendo la riqueza lingüistica de Colombia", permitieron no sólo aplicar con éxito los conceptos trabajados en el aula de clase sino establecer las formas de comportamiento lingüistico de varias regiones del pais.
} 
motivarlo y fortalecer sus destrezas de trabajo en grupo, al mismo tiempo que aprende y se inicia en la investigación, la estrategia que proponemos sería plenamente efectiva. Permite que los estudios traspasen los muros institucionales, fortalece los aprendizajes, refuerza los lazos de amistad, promueve el conocimiento compartido, provoca cuestionamientos, genera curiosidad, fomenta la creatividad, anima la criticidad, aumenta el interés por la lengua y cultura propias, produce ciencia.

La gramática en contexto para nosotros, se convertiría en uno de los modos más efectivo de aprender, explorar, investigar y forjar conocimiento, de una forma práctica y agradable. Su aplicación, necesita maestros comprometidos con el saber y su búsqueda, inteligentes, críticos, organizados, adaptables, propositivos, coherentes, innovadores y recursivos, que sirvan como facilitadores del fortalecimiento de las capacidades intelectuales del aprendiz.

\section{Fundamentos: ¿por qué la gramática?}

La inclusión de la enseñanza gramatical en las aulas escolares o universitarias surge, ante todo, de las disposiciones gubernamentales a través del Ministerio de Educación Nacional. Por ello tenemos que, para el primer grupo de ambientes, los de la escuela, tanto en los lineamientos (1998:28) como en los estándares curriculares (2002:22), se refieren unas "propuestas curriculares que se organizan en función de la interestructuración de los sujetos, la construcción colectiva e interactiva de los saberes y el desarrollo de competencias", con el objeto de formar estudiantes que "aprendan a usar el lenguaje para expresarse con autonomía, comunicarse efectivamente, saber relacionarse con los demás y desarrollar el pensamiento".

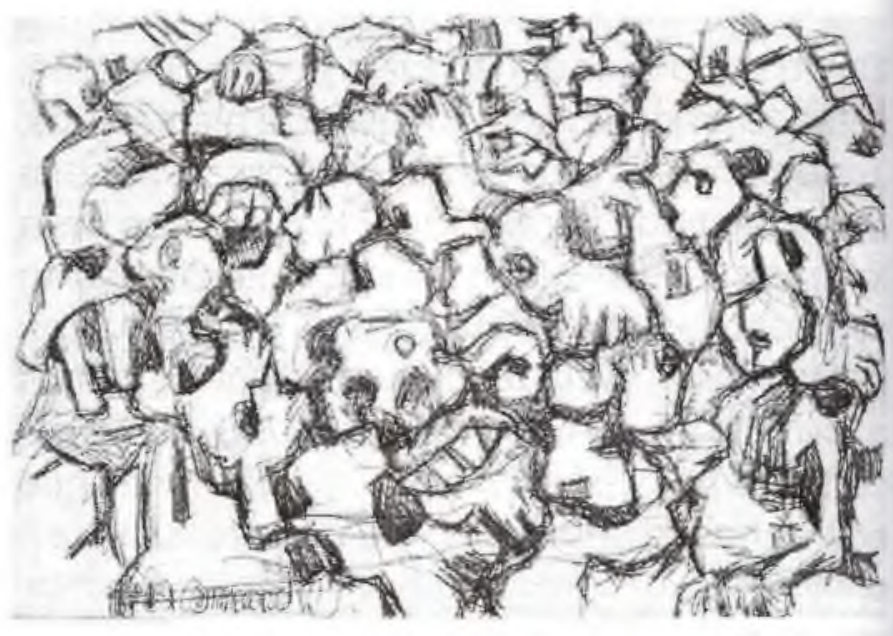

De lo anterior se desprende que, para los objetivos ministeriales, la gramática es una competencia que conforma, junto con las competencias textual, semántica, pragmática, literaria y poética, una competencia significativa. Nuestra competencia gramatical -o sintáctica entonces, refiere las reglas sintácticas, morfológicas, fonológicas y fonéticas que rigen la producción de los enunciados lingüísticos. No se trata pues de algo meramente formalista ya que establece la idea de abordar el estudio del fenómeno lingüístico como resultado de procesos mentales asociados con componentes pragmáticos y semióticos que el hablante pone en juego cada vez que comunica en un contexto específico. Debemos aplicar entonces, un estudio gramatical con características de análisis del discurso, sin estudiar oraciones aisladas o en abstracto sino vistas en su relación con otras dentro de un mismo texto o conversación.

Ahora bien, para el segundo grupo de ambientes, los universitarios, debemos revisar las dos maneras de ver la gramática. Una, como instrumento que coadyuva en los procesos de producción/comprensión del sistema -propio de programas que no tienen el lenguaje como cimiento, y otra, como objeto de estudio -propio de programas de formación de investigadores $y$ docentes de lenguas.

Para quienes tienen los estudios del lenguaje 
como instrumento, citamos a Peña ${ }^{85}$ (2008:89) quien aconseja abordar la gramática en la universidad, ya no con la especificidad de la educación media, sino para entenderla como el principio que organiza la lengua y que demanda estudiarla según sus usos sociales y las principales formas discursivas, de acuerdo con las intenciones del hablante y las situaciones concretas en las que ocurren las interacciones comunicativas. Para el autor, esta forma permitirá que el futuro profesional, desarrolle adecuadamente las competencias críticas, profesionales, sociales, semióticas y culturales que necesitará para la vida.

Para quienes tienen al lenguaje como objeto de estudio, en consonancia con lo anterior tenemos, de un lado, al ICFES (2010:16) quien, como órgano regulador de la educación, espera que el profesional docente en lengua castellana tenga "conocimiento y dominio acerca de la didáctica propia del área [...] que le sirva como base para enfrentar los problemas de la enseñanza y el aprendizaje de la lengua materna en nuestro medio. Este dominio permite llevar a cabo una aproximación pertinente entre la acción y la reflexión, a través del proceso formativo y en su práctica profesional docente". Se busca pues, un docente con gran formación académica y con habilidades que le permitan vincular coherentemente, el logos con la praxis.

Del otro lado está la especificidad universitaria. De manera general, los programas de Licenciatura en lenguas tienen entre sus objetivos la Enseñanza-aprendizaje y la Comunicación que exigen un trabajo crítico, centrado en los discursos contextualizados. En concordancia con estas ideas tenemos a Mahecha (2009:28), quien especifica que "el profesor de idiomas debe comprender la estructura, la función y el funcionamiento del sistema lingüístico, detallando los diferentes planos que lo componen. Debe conocer las implicaciones socioculturales de la lengua, fruto del proceso de representación mental de las comunidades". Entendemos pues que, más allá de analizar y conocer la estructura formal de la lengua -que se lograría con la gramática, hay que ubicarla en un contexto de ejercicio, en una realidad que le permitirá al, ahora investigador, entender no sólo el proceso de significación sino el marco ideológico-social de la comunicación, e ir más allá con criticidad y creatividad.

Hemos de resaltar, para terminar, que en estas orientaciones se pone al contexto como elemento fundamental del estudio gramatical. $Y$ no sólo refiere el contexto lingüístico propio del acto comunicativo en sí, sino al extralingüistico que es el generador de ese acto. En nuestra propuesta iremos más allá pues, al abordar la lengua como un elemento vivo, entramos en contacto con los productores concretos de la comunicación, con su cultura, con su idiosincrasia. Es un acercamiento que nos permitirá, al mismo tiempo que estudiar, identificarnos y reconocer al otro como poseedor de un bagaje socio-semiótico que vale la pena explorar.

\section{Fundamentos: ¿qué respalda la propuesta?}

Una estrategia basada en la gramática en contexto tiene cimientos teóricos fuertes en lo cognitivo, en donde hay un marcado interés por los procesos de aprendizaje y las formas de procesamiento de la información. Esta vía nos condujo a Gardner (1999), quien nos llevó a

${ }^{85} \mathrm{El}$ documento hace parte del trabajo realizado por el autor en el marco del Comité Consultivo para la Definición de Estándares y Evaluación de Competencias Básicas en la Educación Superior, conformado por iniciativa del Ministerio de Educación Nacional. 
comprender que la forma de enseñar, para nuestro caso, la gramática del español, ha tenido una práctica un tanto mal enfocada.

Si tomamos como base la idea de que la inteligencia ${ }^{86}$ no es sólo una sino que es parte de unas competencias intelectuales que están en mayor o menor grado en todos los hombres, tenemos que buscar la forma más efectiva de lograr su desarrollo en el nivel más alto posible. Para lograr este objetivo el autor (1999:133) nos dice: "aunque el conocimiento escolar a menudo está disociado de los contextos del mundo real, es en los contextos ricos, especificos de una situación concreta donde las inteligencias se desarrollan por lo general de una forma productiva".

Podemos deducir que Gardner nos propone unos aprendizajes basados, ya no sólo en realidades contextuales -al estilo montaje escénico, sino desarrollados dentro del contexto con el propósito de alcanzar, además del desarrollo intelectual, la productividad que se constituye como resultado invaluable de las prácticas cognitivas en y para la comunidad. Esta intención se desarrolla completamente con las concepciones constructivistas con las que se relacionan, de una forma u otra, la pedagogía por proyectos, la enseñanza dialogante y el aprendizaje cooperativo.

La afinidad es notoria con Garzón y Vivas (1999:2) para quienes "el conocimiento no se recibe pasivamente ni es una copia de la realidad, sino que es una construcción del sujeto, a partir de la acción en su interacción con el mundo y con otros sujetos". Esta forma de aprender parte de conocimientos y experiencias previas que reacomodan lo nuevo, involucrando actitudes, expectativas y motivaciones, en un proceso dinámico que reestructura la realidad al interactuar en y con ella. Allí el docente es un facilitador de la construcción cognitiva que emerge de una realidad sensopercibida.

De la misma forma, y como se mencionó antes, en esta idea se encauzan la pedagogía por proyectos, la enseñanza dialogante y el aprendizaje cooperativo. En conjunto, los aprendizajes se estructuran como una experiencia participativa, activa y colaborativa, con un alto grado de motivación e implicación del aprendiz. Se presenta un desarrollo global del aprendizaje canalizado por la investigación, y un manejo de contenidos transversales cuando se despliega un plan de trabajo concertado profesor y estudiante en diálogo, en el que se abordarán temas de interés que surjan de la necesidad y del entorno del aprendiz. Se corrobora entonces, lo que dice Rojas (2001:6): "el aprendizaje se basa en experiencias previas vividas en el ejercicio de la libertad. Busca el desarrollo de habilidades para transformar la realidad"

\section{Fundamentos: ¿por qué el trabajo de campo?}

Cuando hablamos de trabajo de campo en ciencias humanas, hacemos conexión directa con las áreas de los estudios sociales: geografia, historia, antropología, sociología. Muy pocas personas creen que un trabajo de este tipo puede ser útil en la enseñanza y la investigación sobre el lenguaje y la lengua, sin embargo, como dijimos más arriba, ha sido la experiencia la que nos ha llevado por estos caminos y gracias a ella, ahora nos aventuramos a defender la idea como propuesta pedagógica y forma de investigación.

\footnotetext{
${ }^{86}$ Tomamos inteligencia como la explica Gardner (1999:33): "una inteligencia implica la habilidad necesaria para resolver problemas o para elaborar productos que son de importancia en un contexto cultural o en una comunidad determinada".
} 
Para nosotros, el trabajo gramatical sin trabajo de campo es bastante equiparable al mito de la Caverna de Platón. Algunas formas de enseñanza de la gramática no alcanzan siquiera al equivalente de las sombras de la citada alegoria; serian como muñequitos de barro creados por otros para dar idea a quienes los ven. Es una gramática descontextualizada. Luego, existen las formas que empiezan a imitar la realidad, equivalentes ahora sí a las sombras proyectadas en la pared de la caverna, sin embargo, no son la realidad en sí. Es una gramática semicontextualizada. Finalmente estarían las formas que muestran la realidad, equivalentes a la realidad fuera de la caverna. Es la gramática en contexto.

Apoyando nuestras ideas, citamos a Pulgarin y otros (1998), para quienes las salidas pedagógicas son, ante todo, una estrategia que suscita la interpretación y la comprensión concretas de la realidad de forma amena y vivencial. Por ellas, los estudiantes pueden manejar de forma general los conceptos y acercarse crítica y propositivamente a los problemas que se presentan en el entorno, de los que se apropian mediante la observación. Para Moreno y otros (2011:15), más que una estrategia, la salida de campo es un escenario pedagógico en donde "se recupera el "mundo de la vida" y se posibilita el diálogo con las ontologias regionales, colocando como centro del proceso educativo la acción intersubjetiva en contra de la imposición de saberes hegemónicos".

Es así que los estudiantes se apropian de una realidad que se vería bien lejana de ser estudiada sólo en el aula de clase. Esto sin olvidar dos factores fundamentales que entran a participar cuando se hace trabajo contextualizado: la motivación y la relación docente-estudiante. Para Cely y otros (2008:104), en el primero debemos tener en cuenta la distancia entre el aprendiz y el conocimiento; si están muy lejos, el objeto se complejiza y se hace de muy difícil acceso; si están muy cerca, el objeto se hace tan familiar que el reto desaparece y se hace muy fácil de obtener. Uno y otro provocan desinterés y desmotivación, lo ideal es la mediación.

Para el segundo, Cely y otros, basados en Coll (2002) establecen que "una buena relación y una excelente comunicación permitirán que los logros propuestos se alcancen con menos dificultades. La aceptación, la confianza mutua y el respeto posibilitan las relaciones afectuosas, brindan seguridad y facilitan la formación de una autoimagen ajustada y positiva". El docente se convierte entonces, no sólo en facilitador, como ya habiamos dicho, sino en acompañante del proceso.

\section{Fundamentos: ¿cómo funcionaría?}

Antes introdujimos que la aplicación de la gramática en contexto debe asentarse en la integración de los objetivos educativos y los intereses considerados por los estudiantes; esto se manifiesta en hipótesis sobre el conocimiento deseado y necesidades por abordar. De acuerdo con ello, para Correa y otros (2003:25), el trabajo de aula apunta a "estudiar la lengua desde una perspectiva discursiva y llevar a cabo un acercamiento a los fenómenos gramaticales en que se hacen evidentes: a) las necesidades cognitivas del estudiante; b) el estudio de la gramática desde las exigencias que plantee la construcción de discurso $y, c)$ los aportes que la gramática ofrece para la concreción de los procesos de significación y de comunicación".

Ahora bien, como nuestra propuesta no está proyectada sólo para un momento del curso, sino que debe organizarse y ejecutarse desde el principio, en la tabla esquematizamos muy someramente (no es el objetivo de este ensayo), la que sería su posible organización: 
Contenidos del curriculares.

Se organizarán los contenidos academicos de la competencia uramatical obscrvando sicmprela construecion de la competencia significativa.
Trabajo de campo.

Se in iciará el trabajo conperativo por proyectos escogiendo tha situación problenica que surge de la neesidad y el interes del estudiante.
Se establexerá el aceremiento compresivo a los contenidos tacilitando daceso a la intornación sobre los contenidos basies y las temiltas pu trabajar.
Se hará una construción conceptual previa en funcion de la situación problemica: objetives.

hipotesis, antecedentes, fundamentos leiricos...

So llevari a cabo el trabajo de campo que tendrá como objuivo la contastacion de las hipótesis en función de la realidad observada. Aquíse verificaran los aprendizajes al evaluar los resultados de los procesos cognilivos a investigativos llevadox a cabo.

Terminamos esta breve explicación con Tobón (1993) quien asegura que un trabajo coherente en el curso de lengua se fundamentaría en un estudio triádico conformado por los componentes semántico, gramatical y pragmático de la producción discursiva.

Para nuestra propuesta, iniciamos refiriendo el componente gramatical que, para la autora (1993:310) debe dar cuenta de "los procedimientos de linearización que permiten al hablante convertir la configuración semántica en una estructura linear, gracias a procesos post-semánticos de selección del tema de su mensaje, determinación del sujeto del verbo y definición del foco". En ellos, se estudian las estructuras formales del predicador, las categorías -léxicas y sintácticas, los marcadores que dan cuenta de las diversas funciones de los casos semánticos, las marcas que permiten inferir relaciones formales entre los elementos del enunciado, y los marcadores de relación frásica en el discurso (referencias fóricas y deixis).

Sin embargo, un estudio del discurso fundamentado sólo con la parte formal, sería ilógico y no concordaría con la propuesta y la exposición que hemos hecho. Por eso debemos citar de nuevo a Tobón (1993:311) quien nos sugiere tener en cuenta las partes semántica y pragmática del enunciado.

En la semántica, se aborda el procesamiento de la información sensopercibida en estructuras mentales resultantes de las asociaciones conceptuales, los conceptos-conocidos como casos semánticos, constituidos por rasgos distintivos que determinan y explican los usos contextuales. En la pragmática, se hacen relevantes aspectos como la norma social que rige las interacciones y la conducta verbal de los individuos, la adecuación de los usos verbales a los comportamientos sociales, las reglas de uso que garantizan eficiencia en producción y comprensión de los procesos discursivos, la lógica de la lengua y la forma que permite distinguir actos de habla en función de la intención comunicativa.

\section{Ventajas y posibles desventajas}

El estudio de la gramática en contexto plantea muchas ventajas que enumeramos arriba y que 


\section{PAIDEAaSurcolombiana 17}

ahora acompañamos con su caracterización:

Permite que los estudios traspasen los muros institucionales; se amplía la concepción de clase al dinamizar los procesos en los diversos ambientes.

Fortalece los aprendizajes; al relacionar saber con hacer, se confronta la teoría con la práctica verificando y construyendo saberes, además de integrar y profundizar el conocimiento.

Refuerza los lazos de amistad; al compartir espacios e intereses se establece el predominio de los afectos.

Promueve el conocimiento compartido; por el aprendizaje cooperativo, el trabajo grupal permite la construcción colectiva.

Genera curiosidad; cuando se explora el contexto, se despierta el deseo de saber más sobre sus componentes y los comportamientos que llevan a cabo.

Provoca cuestionamientos; cuando se busca la respuesta a unos, surgen otros, ello hace parte del espiral holístico de la investigación. Anima la criticidad; cuando se conoce la realidad y lo que deberia ser, se despierta el espiritu crítico haciendo juicios sobre ella.

Fomenta la creatividad; el conocer y ser crítico propicia soluciones innovadoras a las problemáticas trabajadas o surgidas durante el trabajo.

Aumenta el interés por la lengua y cultura propias; cuando el trabajo de campo se centra en los contextos nacionales y regionales.

Produce ciencia; cuando se estimula el trabajo investigativo, hay una aproximación conceptual previa, formulación de hipótesis y verificación.
Ahora lo negativo. Cuando se planean estudios de campo, puede ( $n$ ) encontrarse:

Fallas en la organización y metodología del docente.

" Falta de disponibilidad de tiempo y de presupuesto.

Exceso de trámites administrativos y con las familias.

- Desmotivación estudiantil.

Comportamientos inadecuados.

Peligros en carretera.

Delincuencia.

\section{A manera de conclusión}

Pudimos ver en las líneas anteriores todo el constructo teórico, el funcionamiento y los pros y contras de nuestra gramática en contexto. Para nosotros es una estrategia bastante funcional que hará que el estudio y la investigación, sobre todo en el ámbito colombiano, no escapen de la mente del docente en formación. Así, todo lo que éste lea, explore, haga e innove, servirá para seguir construyendo la red cognitiva que fortalecerá el proceso educativo en el país.

Estamos seguros de que la excelencia empieza en el aula de clase y de que todo cuanto se construya en ella se puede conectarse con las experiencias de campo, logrando que la universidad se fortalezca no sólo en el ámbito investigativo, sino en el académico y productivo. 


\section{Bibliografía}

COLL, César (comp.) (2002). El constructivismo en el aula. Barcelona: Grao

CORREA, José Ignacio (coord.) (2003). Estándares básicos de competencias del lenguaje. Formar en lenguaje: apertura de caminos para la interlocución. Bogotá: Ministerio de educación.

GARDNER, Howard (1999). Inteligencias múltiples: la teoria en la práctica. Barcelona: Paidós

. (2001). Estructuras de la mente: la teoría de las inteligencias múltiples. Bogotá: Fondo de Cultura Económica

GARZÓN, Carlos y VIVAS, Mireya (1999). Una didáctica constructivista en el aula universitaria. En: Educere, Revista venezolana de educación. Año 3. No. 5. Mérida: Universidad de los Andes [citado el 30-11-2011]. Disponible en internet en: $<$ http://www.saber.ula.ve/handle/123456789/ 4323>

INSTITUTO COLOMBIANO PARA LA EVALUACIÓN DE LA EDUCACIÓN. Orientaciones para el examen de Estado de calidad de la educación superior, Saber pro: Licenciatura en educación básica con énfasis en humanidades y lengua castellana. Bogotá [citado el 25-11-2011]. Disponible en internet en: $<$ http://www.icfes.gov.co/

MAHECHA B., Miguel Ángel (2009). ¿Para qué enseñamos lingüistica? En: Paidea Surcolombiana N0.14. Mayo. Neiva: Facultad de Educación, Universidad Surcolombiana. p. 21-33

MIER I., Freddy Alberto (2010). El discurso de los textos de español: un análisis crítico. En: Congreso nacional de estudios del discurso (6:
13-15 octubre: Cartagena de Indias, Bolivar). Asociación latinoamericana de estudios del discurso.

MINISTERIO DE EDUCACIÓN NACIONAL (1998). Lineamientos curriculares: Lengua castellana. Bogotá.

(2002) Estándares básicos de matemáticas y lenguaje: Educación básica y media. Bogotá

MORENO U., Nubia, Rodríguez P., Liliana y SánchezA., Jorge (2011). Salida de campo: se hace escuela al andar. Bogotá, Universidad Distrital Francisco José de Caldas

PEÑA BORRERO, Luis Bernardo (2008). La competencia oral y escrita en la educación superior [en línea]. Bogotá: Ministerio de Educación [citado el 28-11-2011]. Disponible en internet en: <htp://www.mineducacion.gov.co/ 1621/article-189357.html>

PORLÁN, Rafael. (1995). Constructivismo y Escuela. Madrid: Diada

PULGARIN SILVA, María Raquel (1998). La salida pedagógica estrategia fundamental en el aprendizaje de las ciencias sociales. Medellín: Zuluaga

ROJAS VELÁSQUEZ, Freddy (2001). Enfoques sobre el aprendizaje humano [en línea]. Caracas: Universidad Simón Bolívar, Departamento de ciencia y tecnologia del comportamiento [citado el 28-11-2011]. Disponible en internet en: <http:/ /repositorio.oui-iohe.org/dspace/bitstream/ $123456789 / 1459 / 1 /$

TOBÓN DE CASTRO, Lucía (1993). Propuesta de un proyecto lingüistico para el estudio del español como lengua propia. En: Thesaurus. Tomo XLVIII. No. 2. Bogotá: Instituto Caro y Cuervo 